\title{
The host of GRB 060206: kinematics of a distant galaxy
}

\author{
C. C. Thöne ${ }^{1}$, K. Wiersema ${ }^{2}$, C. Ledoux ${ }^{3}$, R. L. C. Starling ${ }^{4}$, A. de Ugarte Postigo ${ }^{3,5}$, A. J. Levan ${ }^{6}$, J. P. U. Fynbo ${ }^{1}$, \\ P. A. Curran ${ }^{2}$, J. Gorosabel ${ }^{5}$, A. J. van der Horst ${ }^{2}$, A. Llorente ${ }^{7}$, E. Rol ${ }^{4}$, N. R. Tanvir ${ }^{4}$, P. M. Vreeswijk ${ }^{1,3}$, \\ R. A. M. J. Wijers ${ }^{2}$, and L. J. Kewley ${ }^{8}$
}

1 Dark Cosmology Centre, Niels Bohr Institute, University of Copenhagen, Juliane Maries Vej 30, 2100 Copenhagen, Denmark e-mail: cthoene@dark-cosmology . dk

2 Astronomical Institute "Anton Pannekoek", University of Amsterdam, Kruislaan 403, 1098 SJ Amsterdam, The Netherlands

3 European Southern Observatory, Alonso de Córdova 3107, Vitacura, Santiago 19, Chile

4 Dept. of Physics and Astronomy, University of Leicester, University Road, Leicester LE1 7RH, UK

5 Instituto de Astrofísica de Andalucía, (IAA-CSIC), Apdo. 3004, 18080 Granada, Spain

6 Department of Physics, University of Warwick, Coventry CV4 7AL, UK

7 Herschel Science Operations Center, INSA, ESAC, European Space Agency, Villafranca del Castillo, PO Box 50727, 28080 Madrid, Spain

8 University of Hawaii, 2680 Woodlawn Drive, Honolulu, HI 96822, USA

Received 25 Aout 2007 / Accepted 28 May 2008

\section{ABSTRACT}

\begin{abstract}
Context. GRB afterglow spectra are sensitive probes of interstellar matter along the line-of-sight in their host galaxies, as well as in intervening galaxies. The rapid fading of GRBs makes it very difficult to obtain spectra of sufficient resolution and $\mathrm{S} / \mathrm{N}$ to allow for these kinds of studies.

Aims. We investigate the state and properties of the interstellar medium in the host of GRB 060206 at $z=4.048$ with a detailed study of groundstate and finestructure absorption lines in an early afterglow spectrum. This allows us to derive conclusions on the nature and origin of the absorbing structures and their connection to the host galaxy and/or the GRB.

Methods. We used early (starting $1.6 \mathrm{~h}$ after the burst) WHT/ISIS optical spectroscopy of the afterglow of the gamma-ray burst GRB 060206 detecting a range of metal absorption lines and their finestructure transitions. Additional information is provided by the afterglow lightcurve. The resolution and wavelength range of the spectra and the bright afterglow have facilitated a detailed study and fitting of the absorption line systems in order to derive column densities. We also used deep imaging to detect the host galaxy and probe the nature of an intervening system at $z=1.48$ seen in absorption in the afterglow spectra.

Results. We detect four discrete velocity systems in the resonant metal absorption lines, best explained by shells within and/or around the host created by starburst winds. The finestructure lines have no less than three components with strengths decreasing from the redmost components. We therefore suggest that the finestructure lines are best explained as being produced by UV pumping from which follows that the redmost component is the one closest to the burst where $\mathrm{N}$ V was detected as well. The host is detected in deep $H$ ST imaging with $F 814 W_{A B}=27.48 \pm 0.19$ mag and a $3 \sigma$ upper limit of $H=20.6$ mag (Vega) is achieved. A candidate counterpart for the intervening absorption system is detected as well, which is quite exceptional for an absorber in the sightline towards a GRB afterglow. The intervening system shows no temporal evolution as claimed by Hao et al. (2007, ApJ, 659, 99), which we prove from our WHT spectra taken before and Subaru spectra taken during those observations.
\end{abstract}

Key words. gamma rays: bursts - galaxies: abundances - cosmology: observations

\section{Introduction}

In recent years, GRBs have been detected out to very high redshifts with four at $z>5$ currently (Jakobsson et al. 2006a; Cenko et al. 2006; Ruiz-Velasco et al. 2007; Haislip et al. 2006; Kawai et al. 2006). Theory and observations suggest that $\sim 10 \%$ of Swift-detected gamma-ray bursts (GRBs) will originate at redshifts $z \gtrsim 5$ and $\sim 3 \%$ at redshifts $z \gtrsim 6$ (Bromm \& Loeb 2006; Daigne et al. 2006; Tanvir \& Jakobsson 2007). Furthermore, the observed flux is not expected to fade significantly with increasing redshift as time dilation effects lead to earlier observations of the afterglow in the GRB restframe. GRBs should therefore be detectable out to $z \gtrsim 10$ (Ciardi \& Loeb 2000), making them the most distant observable objects in the Universe. Indeed, some of the most distant GRB afterglows were also among the most luminous ones observed: e.g. GRB 050904, $z=6.3$ (Kawai et al. 2006) and GRB 060206, $z=4.048$, presented here (see also Monfardini et al. 2006). As such, these events can teach us something about galaxy evolution and the early Universe: the occurrence of GRBs in starforming regions in high-redshift galaxies allows us to probe sightlines to these galaxies with high spectral resolution, by exploiting the brightness and simple spectral slope of GRB afterglows (e.g. Prochaska et al. 2007; D'Elia et al. 2007; Vreeswijk et al. 2007).

Through studies of absorption lines in the spectra of GRB afterglows, it is possible to determine properties of these distant galaxies, which are otherwise hard to obtain. One key piece of information that can be derived from resonant metal absorption lines is the metallicity along the line-of-sight towards the GRB in the host galaxies. These restframe-UV transitions are shifted into the optical regime above $z \sim 2$, hence are observable with ground-based telescopes. The line-of-sight metallicities derived from afterglow spectroscopy are higher than the mean QSO absorber metallicity as a function of redshift (Fynbo et al. 2006; Prochaska et al. 2007). This might, however, only be caused by a selection effect, because the sightlines probed by QSOs and 
GRBs are probably different (Fynbo et al. 2008; Prochaska et al. 2007, see Fig. 1). At redshifts below $z \sim 1$ GRB hosts have lower metallicities than a sample of blue, star-forming galaxies from the SDSS survey (Tremonti et al. 2004). For these nearby galaxies, the metallicities are derived from emission line diagnostics and reflect the metallicity of the HII regions within these galaxies (Savaglio 2006).

QSO absorption systems have been used for a long time to study distant galaxies in a similar way as GRBs, but an association of the absorbing system with a galaxy is not always possible due to the brightness of the QSO itself. Furthermore, QSO sightlines seem to probe the less dense outer regions of galaxies, whereas GRBs often reside inside a galaxy and their sightlines also allow us to probe the inner parts of the galaxy and the immediate environment of the burst (see e.g. Fig. 1 in Prochaska et al. 2007). In all GRB spectra with the right wavelength coverage and high enough $\mathrm{S} / \mathrm{N}$ and resolution, finestructure lines such as $\mathrm{Si} \mathrm{II}^{*}, \mathrm{Fe}_{\mathrm{II}}^{*}$, O I* (Prochaska et al. 2007) and even higher levels have been detected (e.g. Vreeswijk et al. 2007). These lines are produced by collisional excitation or indirect UV pumping of the levels from the strong UV radiation field of the GRB and may therefore be closely connected to the GRB itself. In QSO absorbers, only finestructure lines of C I and C II have been detected so far (Silva \& Viegas 2002, and references therein), but no other finestructure transitions such as $\mathrm{Si}$ II* or Fe II*.

In our first paper on the dataset presented here (Fynbo et al. 2006, hereafter Paper I), we showed initial results of the WHT and NOT spectra of the afterglow of GRB 060206, focusing on the metallicity that we compared to a large sample of QSO absorber metallicities and the sample of known GRB afterglow metallicities. In this second paper we analyze the full set of absorption lines and add a more detailed analysis of the properties of the host from the photometric detection of a possible host galaxy and broadband afterglow properties. The paper is organized as follows. In Sect. 2 we present the spectroscopy of the GRB afterglow and the observations of the host galaxy. In Sect. 3 we describe the absorption line measurement method and results. In Sect. 4 we analyze the discrete velocity components in the host through the detected (finestructure) lines and the line-ofsight extinction. We present the properties of the host galaxy in emission in Sect. 5 through late deep imaging. In Sect. 6 we discuss the absorption line properties of the intervening absorbers, and evaluate the possibility that a galaxy close to the afterglow location is responsible for one of the intervening absorber systems. Throughout this paper we use the cosmological parameters $H_{0}=70 \mathrm{~km} \mathrm{~s}^{-1} \mathrm{Mpc}^{-1}, \Omega_{\mathrm{M}}=0.3$, and $\Omega_{\Lambda}=0.7$.

\section{Observations}

\subsection{Spectroscopy}

We make extensive use in this paper of the dataset described in Paper I, to which we refer for details on the observations and data reduction. For clarity, we reiterate here the important steps. We obtained a medium-resolution spectrum with the Intermediatedispersion Spectroscopic and Imaging System (ISIS) on the $4.2 \mathrm{~m}$ William Herschel Telescope (WHT) on 2006 February 6 , starting $1.61 \mathrm{~h}$ after the burst. We took two spectra with exposure times of $900 \mathrm{~s}$ each, separated only by the read-out time of the CCD. The spectrograph has a blue and a red arm, providing FWHM resolutions of 1.68 and $0.82 \AA$, respectively. A redshift of $z=4.04795 \pm 0.00020$ was determined from several absorption lines within the host (Paper I). At this redshift, the WHT spectra cover the rest frame wavelength range of approximately 753-1030 and 1228-1387 A for the blue and red arms, respectively. As the Ly $\alpha$ line falls into the gap between the two arms, we used the column density derived from the NOT spectra (Paper I) for deriving metallicities. The $\mathrm{S} / \mathrm{N}$ ratios of the spectra are high, strongly aided by the sudden, strong rebrightening of the afterglow just prior to and during the spectroscopic observations reaching a maximum of $R \sim 16.4$ mag (Woźniak et al. 2006).

Furthermore, we obtained $8 \times 1800$ s low-resolution $(R \sim 300)$ spectra from the SUBARU archive Baba et al. (2002) from about 6 to $10 \mathrm{~h}$ after the burst. We reduced the spectra with standard tasks in IRAF.

The optical afterglow of this burst proved bright with $R \sim 18.1 \mathrm{mag}$ after 1 day, and owing to its fortunate location on the sky (RA 13:31:43, Dec +35:03:03 (J2000)), it was observed at a wide range of northern observatories (e.g. Woźniak et al. 2006; Monfardini et al. 2006; Stanek et al. 2007). A detailed discussion of photometric afterglow observations and the lightcurve physics can be found in Curran et al. (2007). We used these observations, together with Swift XRT data, to analyze the broadband optical to X-ray extinction (see Sect. 3).

\subsection{Imaging}

We observed the location of the afterglow on February 16, 2007, over a year after the burst, using the GMOS-N instrument on Gemini-North with the aim of detecting the host and intervening absorber galaxies in emission. Observations were performed in the GMOS $r^{\prime}$ band, which has a similar shape and throughput as the SDSS $r^{\prime}$ band. The dataset consists of $9 \times 300 \mathrm{~s}$ exposures with a seeing of $\sim 1$ arcsec and an airmass of $\sim 1$.1. Reduction was done using the Gemini package in IRAF and the photometry was calibrated against archival SDSS observations of the GRB 060206 field (Cool et al. 2006). For the combined image we derive a $3 \sigma$ upper limit of $r_{A B}^{\prime}=26.3$ which was obtained by examining the standard deviation of 50 blank apertures placed at random positions on the image.

We also observed the GRB field in the near-infrared on June 3, 2007 using the Omega2000 instrument on the $3.5 \mathrm{~m}$ telescope at the Calar Alto Astronomical Observatory (CAHA). Observations consisting of $50 \times 60 \mathrm{~s}$ were performed in the $H$-band with a limiting magnitude of $H=20.6 \mathrm{mag}$ (Vega). GRB 060206 was finally observed with $H S T$ on 25 November 2006, using the ACS/WFC and F814W filter under the program 10817 (PI H.-W. Chen), which are now publicly available from the HST archive. Eight dithered exposures were obtained with a total exposure time of $9886 \mathrm{~s}$. We processed these data through the standard multidrizzle pipeline, setting the linear drop size (pixfrac) to one, and the final output scale to 0.'033 per pixel. Performing relative astrometry between our early NOT observations (Fynbo et al. 2006; Curran et al. 2007) allows us to place the afterglow on this image with an accuracy of $\sim 0$. $^{\prime} 05$.

\section{Extinction along the line-of-sight}

To obtain limits on the absorption along the line of sight to GRB 060206, we fit the near-infrared to X-ray spectral energy distribution (SED) in count space (Starling et al. 2007), and using the metallicity as determined through the sulphur lines of $[\mathrm{S} / \mathrm{H}]=-0.86$. The SED is created using RJHK photometry and an X-ray spectrum from Swift XRT (Gehrels et al. 2004; Burrows et al. 2005), centered at $\sim 2.8 \mathrm{~h}$ after burst, detailed in Curran et al. (2007). We note that this is close in time to our 
Table 1. Velocity decomposition and Voigt profile fitting of the different components in the host galaxy absorption systems.

\begin{tabular}{|c|c|c|c|c|c|c|c|c|c|}
\hline ID & $\begin{array}{l}\lambda_{\text {rest }} \\
{[\AA]}\end{array}$ & comp. & $\begin{array}{l}\lambda_{\text {obs }} \\
{[\AA]}\end{array}$ & $\begin{array}{l}v_{\text {abs }} \\
{\left[\mathrm{km} \mathrm{s}^{-1}\right]}\end{array}$ & $\begin{array}{r}\log N \\
{\left[\mathrm{~cm}^{-2}\right]}\end{array}$ & $\begin{array}{l}b_{\text {turb }} \\
{\left[\mathrm{km} \mathrm{s}^{-1}\right]}\end{array}$ & $\begin{array}{l}\log N_{\text {tot }} \\
{\left[\mathrm{cm}^{-2}\right]}\end{array}$ & {$[\mathrm{X} / \mathrm{H}]$} & $\begin{array}{l}\text { blended } \\
\text { with }\end{array}$ \\
\hline \multirow[t]{4}{*}{$\mathrm{S}_{\text {II }}$} & 1250.584 & 1 & 6316.51 & 169 & $14.51 \pm 0.11$ & 15 & $15.13 \pm 0.05$ & $-0.86 \pm 0.09$ & \\
\hline & & 2 & 6314.41 & 69 & $14.52 \pm 0.11$ & 25 & & & \\
\hline & & 3 & 6312.71 & -11 & $14.71 \pm 0.07$ & 25 & & & \\
\hline & & 4 & 6308.13 & -228 & $14.27 \pm 0.19$ & 15 & & & \\
\hline \multirow[t]{4}{*}{ S II } & 1253.811 & 1 & 6332.81 & 169 & $14.51 \pm 0.11$ & 15 & $15.13 \pm 0.05$ & & \\
\hline & & 2 & 6330.70 & 69 & $14.52 \pm 0.11$ & 25 & & & \\
\hline & & 3 & 6329.00 & -11 & $14.71 \pm 0.07$ & 25 & & & \\
\hline & & 4 & 6324.41 & -228 & $14.27 \pm 0.19$ & 15 & & & \\
\hline \multirow[t]{4}{*}{ S II } & 1259.519 & 1 & 6361.64 & 169 & $14.51 \pm 0.11$ & 15 & $15.13 \pm 0.05$ & & Si III 1260 \\
\hline & & 2 & 6359.52 & 69 & $14.52 \pm 0.11$ & 25 & & & $\mathrm{Si} \mathrm{III}_{1260}$ \\
\hline & & 3 & 6357.81 & -11 & $14.71 \pm 0.07$ & 25 & & & $\mathrm{Si}_{\mathrm{II}} 1260$ \\
\hline & & 4 & 6353.20 & -228 & $14.27 \pm 0.19$ & 15 & & & \\
\hline \multirow[t]{6}{*}{ Si II } & 1260.4221 & 1 & 6366.20 & 169 & $14.41 \pm 0.09$ & 15 & $15.23 \pm 0.04$ & $-1.08 \pm 0.07$ & \\
\hline & & $1 \mathrm{a}$ & 6365.28 & 127 & $12.64 \pm 0.13$ & 20 & & & \\
\hline & & $2 *$ & 6363.70 & 51 & $14.66 \pm 0.05$ & 25 & & & \\
\hline & & 3 & 6362.37 & -11 & $14.79 \pm 0.07$ & 25 & & & \\
\hline & & $3 a$ & 6361.19 & -67 & $13.71 \pm 0.08$ & 20 & & & \\
\hline & & 4 & 6357.76 & -228 & $14.52 \pm 0.08$ & 15 & & & \\
\hline \multirow[t]{6}{*}{ Si II } & 1304.3702 & 1 & 6588.18 & 169 & $14.41 \pm 0.09$ & 15 & $15.23 \pm 0.04$ & & OI* \\
\hline & & $1 \mathrm{a}$ & 6387.22 & 127 & $12.64 \pm 0.13$ & 20 & & & OI* \\
\hline & & $2 *$ & 6585.59 & 51 & $14.66 \pm 0.05$ & 25 & & & OI* \\
\hline & & 3 & 6584.21 & -11 & $14.79 \pm 0.07$ & 25 & & & \\
\hline & & $3 a$ & 6583.00 & -67 & $13.71 \pm 0.08$ & 20 & & & \\
\hline & & 4 & 6579.44 & -228 & $14.52 \pm 0.08$ & 15 & & & \\
\hline \multirow[t]{5}{*}{ O I } & 1302.1685 & 1 & 6577.06 & 169 & $16.02 \pm 0.53$ & 15 & $16.33 \pm 0.29$ & $-1.16 \pm 0.31$ & \\
\hline & & 2 & 6574.86 & 69 & $15.72 \pm 0.24$ & 25 & & & \\
\hline & & 3 & 6573.10 & -11 & $15.52 \pm 0.47$ & 25 & & & \\
\hline & & $3 a$ & 6571.88 & -67 & $14.91 \pm 0.13$ & 20 & & & \\
\hline & & 4 & 6568.33 & -228 & $15.14 \pm 0.18$ & 15 & & & \\
\hline \multirow[t]{6}{*}{$\mathrm{C}_{\mathrm{II}}$} & 1334.5323 & 1 & 6740.52 & 169 & $16.40 \pm 0.39$ & 15 & $>16.85$ & $>-0.39$ & \\
\hline & & $1 \mathrm{a}$ & 6739.55 & 127 & $13.67 \pm 0.42$ & 20 & & & \\
\hline & & 2 & 6738.28 & 69 & $15.10 \pm 0.40$ & 25 & & & \\
\hline & & 3 & 6736.47 & -11 & $>16.50$ & 25 & & & \\
\hline & & $3 a$ & 6735.22 & -67 & $14.69 \pm 0.18$ & 20 & & & \\
\hline & & 4 & 6731.58 & -228 & $16.09 \pm 0.24$ & 15 & & & \\
\hline $\mathrm{NV}$ & 1238.821 & 1 & 6257.46 & 186 & $13.73 \pm 0.06$ & 25 & $13.73 \pm 0.06$ & - & \\
\hline $\mathrm{N} \mathrm{v}$ & 1242.804 & 1 & 6277.58 & 186 & $13.73 \pm 0.06$ & 25 & $13.73 \pm 0.06$ & & \\
\hline
\end{tabular}

WHT spectra, which were taken at mid-time $1.86 \mathrm{~h}$ after burst. We perform fits using an absorbed single power law, and then a broken power law to model a possible cooling break.

The SED is well fit with a single power law with $\beta=0.93 \pm 0.01$ and $\chi^{2} /$ d.o.f. $=1.05$. A broken power law does not provide a significant improvement in the fit according to the $F$-test. We find no evidence for intrinsic $(z=4.048)$ optical or $\mathrm{X}$-ray extinction above the Galactic values. At this high redshift of $z=4.048$ the metal edges that dominate the X-ray extinction are shifted out of the XRT energy range and are not sensitively probed. The near-infrared and optical data at this redshift, however, probe restframe UV and blue bands, and are therefore sensitive to extinction. We estimate a $3 \sigma$ upper limit for the intrinsic optical/UV extinction of $E(B-V)<0.01$. To derive this we fitted the SED in count space from X-ray to optical simultaneously (as described in Starling et al. 2007) with an LMC, SMC and MW extinction law and increased the value of $E(B-V)$ until $\chi^{2}=1$, corresponding to a $1 \sigma$ deviation. This shows that both the intervening systems and the host velocity systems have low dust content, which can be used to distinguish between different origins of these systems.

\section{Absorption line analysis}

We reported equivalent widths and column densities for a selection of absorption lines in Paper I, with the aim of deriving a metallicity for the line-of-sight within the host, the exact velocity decomposition of the systems, and the finestructure line ratios as a function of velocity. Here, we present a full analysis of all absorption lines in the host galaxy and the intervening systems. The results of the host galaxy systems are presented in Tables 1 and 2 and Figs. 1, 2, and 3. The fits for the intervening system can be found in Table 3 and Fig. 4 .

For the host galaxy with a systemic redshift of $z=4.048$, we detect four main velocity systems at $v_{1}=169 \mathrm{~km} \mathrm{~s}^{-1}$, $v_{2}=69 \mathrm{~km} \mathrm{~s}^{-1}, v_{3}=-11 \mathrm{~km} \mathrm{~s}^{-1}$, and $v_{4}=-228 \mathrm{~km} \mathrm{~s}^{-1}$ which we refer to as systems 1 to 4 . To obtain a better fit, we had to introduce two additional subcomponents for all strong lines 
Table 2. Same as Table 1 for the finestructure lines in the host galaxy system that only have 3 components.

\begin{tabular}{|c|c|c|c|c|c|c|c|c|}
\hline ID & $\begin{array}{l}\lambda_{\text {rest }} \\
{[\AA]}\end{array}$ & comp. & $\begin{array}{l}\lambda_{\text {obs }} \\
{[\AA]}\end{array}$ & $\begin{array}{l}v_{\mathrm{abs}} \\
{\left[\mathrm{km} \mathrm{s}^{-1}\right]}\end{array}$ & $\begin{array}{l}\log N \\
{\left[\mathrm{~cm}^{-2}\right]}\end{array}$ & $\begin{array}{l}b_{\text {turb }} \\
{\left[\mathrm{km} \mathrm{s}^{-1}\right]}\end{array}$ & $\begin{array}{l}\log N_{\text {tot }} \\
{\left[\mathrm{cm}^{-2}\right]}\end{array}$ & $\begin{array}{l}\text { blended } \\
\text { with }\end{array}$ \\
\hline \multirow[t]{5}{*}{ Si II ${ }^{*}$} & 1264.7377 & 1 & 6388.00 & 169 & $14.17 \pm 0.04$ & 15 & $14.38 \pm 0.03$ & \\
\hline & & $1 \mathrm{a}$ & 6387.08 & 127 & $12.89 \pm 0.14$ & 20 & & Si II ${ }_{1265}$ \\
\hline & & 2 & 6385.87 & 69 & $13.72 \pm 0.07$ & 25 & & Si II ${ }_{1265}$ \\
\hline & & 3 & 6384.16 & -11 & $13.41 \pm 0.05$ & 25 & & Si II ${ }_{1265}$ \\
\hline & & $3 a$ & 6382.97 & -67 & $12.73 \pm 0.08$ & 20 & & \\
\hline \multirow[t]{5}{*}{ Si II ${ }^{*}$} & 1265.0020 & 1 & 6389.33 & 169 & $14.17 \pm 0.04$ & 15 & $14.38 \pm 0.03$ & \\
\hline & & $1 \mathrm{a}$ & 6388.41 & 127 & $12.89 \pm 0.14$ & 20 & & Si II ${ }^{*}{ }_{1264}$ \\
\hline & & 2 & 6387.21 & 69 & $13.72 \pm 0.07$ & 25 & & Si II ${ }_{1264}$ \\
\hline & & 3 & 6384.16 & -11 & $13.41 \pm 0.05$ & 25 & & Si II ${ }_{1264}$ \\
\hline & & $3 a$ & 6384.21 & -67 & $12.73 \pm 0.08$ & 20 & & Si II ${ }_{1264}$ \\
\hline \multirow[t]{5}{*}{ Si II ${ }^{*}$} & 1309.2757 & 1 & 6612.95 & 169 & $14.17 \pm 0.04$ & 15 & $14.38 \pm 0.03$ & \\
\hline & & $1 \mathrm{a}$ & 6612.00 & 127 & $12.89 \pm 0.14$ & 20 & & \\
\hline & & 2 & 6610.75 & 69 & $13.72 \pm 0.07$ & 25 & & \\
\hline & & 3 & 6608.98 & -11 & $13.41 \pm 0.05$ & 25 & & \\
\hline & & $3 \mathrm{a}$ & 6607.75 & -67 & $12.73 \pm 0.08$ & 20 & & \\
\hline \multirow[t]{2}{*}{$\mathrm{OI}^{*}$} & 1304.8576 & 1 & 6590.64 & 169 & $14.86 \pm 0.07$ & 15 & $14.89 \pm 0.07$ & \\
\hline & & 2 & 6588.44 & 69 & $13.47 \pm 0.06$ & 25 & & Si II ${ }_{1304}$ \\
\hline \multirow[t]{2}{*}{ OI** } & 1306.0286 & 1 & 6596.55 & 169 & $14.15 \pm 0.09$ & 15 & $14.37 \pm 0.07$ & \\
\hline & & 2 & 6594.36 & 69 & $13.98 \pm 0.12$ & 25 & & \\
\hline \multirow[t]{4}{*}{ C II* } & 1335.6630 & 1 & 6746.23 & 169 & $15.76 \pm 0.16$ & 15 & $15.77 \pm 0.15$ & \\
\hline & & $1 \mathrm{a}$ & 6745.26 & 127 & $12.68 \pm 0.71$ & 20 & & \\
\hline & & 2 & 6743.98 & 69 & $13.92 \pm 0.06$ & 25 & & \\
\hline & & 3 & 6743.98 & -11 & $14.02 \pm 0.06$ & 25 & & \\
\hline \multirow[t]{4}{*}{$\mathrm{C}_{\mathrm{II}}{ }^{*}$} & 1335.7077 & 1 & 6746.46 & 169 & $15.76 \pm 0.16$ & 15 & $15.77 \pm 0.15$ & \\
\hline & & $1 \mathrm{a}$ & 6745.49 & 127 & $12.68 \pm 0.71$ & 20 & & \\
\hline & & 2 & 6744.21 & 69 & $13.92 \pm 0.06$ & 25 & & \\
\hline & & 3 & 6742.40 & -11 & $14.02 \pm 0.06$ & 25 & & \\
\hline
\end{tabular}

(see below), which are at velocities of $v_{1 a}=127 \mathrm{~km} \mathrm{~s}^{-1}$ and $v_{3 a}=-67 \mathrm{~km} \mathrm{~s}^{-1}$. In addition, we find an intervening system at $z=1.47895$ that shows two components at $v_{1}=25 \mathrm{~km} \mathrm{~s}^{-1}$ and $v_{1}=-30 \mathrm{~km} \mathrm{~s}^{-1}$. Zero velocities are chosen arbitrarily as the midpoint of the entire system, as the exact redshift of the host and the intervening galaxies are unknown.

To fit the different components, we used the FITLYMAN package in MIDAS (Fontana \& Ballester 1995). The fitting is, however, complicated by the strong blending and saturation in a number of lines. We therefore used the weak, unsaturated S II $\lambda 1253$ transition as a template for the position of the four main components $v_{1}$ to $v_{4}$. For the Si-lines, OI and C II, and the corresponding finestructure lines, we needed two additional components $1 \mathrm{a}$ and $3 \mathrm{a}$ in the blue wings of the saturated components 1 and 3 to achieve a good fit. To obtain a reasonable fit for the Si II system, we had to put component 2 at a slightly different redshift for Si II with a velocity difference of $\Delta v_{2-2 *}=18 \mathrm{~km} \mathrm{~s}^{-1}$, which is therefore denoted as component $2 *$.

Multiplet transitions were fitted such that for each component all individual transitions have the same column density. This is useful in those cases where one of the transitions is blended with another line to disentangle the contributions from the different elements to one absorption feature. Blended lines were fitted together such as C II ${ }^{*} \lambda 1335.6$ and $\mathrm{C}$ II $* \lambda 1335.7$ and Si II $\lambda \lambda 1264$ and 1265 . In case of the blended lines Si II $\lambda 1260$ and S II $\lambda 1259$, S II was excluded for the common fit of the S II lines. For Si II and $\mathrm{OI}^{*} \lambda 1304$ that were fitted together, the contribution from $\mathrm{OI}^{*}$ is negligible and only 2 components were fitted for the finestructure line and the same for OI**. For the finestructure lines $\mathrm{C}$ II $* \lambda \lambda 1335.6,1335.7$, we fitted 3 components that are not blended with the ground-state transition. The fitted values for the different components are then listed in Table 1 for the resonant transitions and Table 2 for the finestructure lines. The metallicities noted are derived from the total column densities including the ground state and finestructure transitions of the individual ionic species. As solar metallicities, we used the values from Asplund et al. (2004). The velocities of the Doppler parameters are given in rest frame.

As shown in Fig. 1, the resonant transitions of C, S, O, and $\mathrm{Si}$ show four main velocity components, the strong transitions of $\mathrm{C}$, $\mathrm{O}$ and $\mathrm{Si}$ have two additional subcomponents. These subcomponents might also be present in the weaker transitions but simply not detected in those transitions. N v $\lambda \lambda 1238,1242$ is detected in a single velocity component at $v_{1 *}=186 \mathrm{~km} \mathrm{~s}^{-1}$ (see Fig. 3) which is at slightly higher velocity than comp. 1 of the other absorption lines. The finestructure lines (Fig. 2) only show 2 or 3 components which is clearly visible from the only unblended finestructurefinestructure line, Si II* $\lambda 1309$.

For all host component lines, we assume only turbulent broadening and estimate the values to be $b_{\text {turb }}=15 \mathrm{~km} \mathrm{~s}^{-1}$ in the two narrow components 1 and $4,25 \mathrm{~km} \mathrm{~s}^{-1}$ in components 2 and 3 and $20 \mathrm{~km} \mathrm{~s}^{-1}$ in the additional components $1 \mathrm{a}$ and $3 \mathrm{a}$. The values are invoked by the best fits from unblended lines. The intervening system at $z=1.478$ is unblended and the multiplet fits for the Mg II $\lambda \lambda 2796,2803$ and Fe II $\lambda \lambda 2586,2600$ doublets give consistent results. 


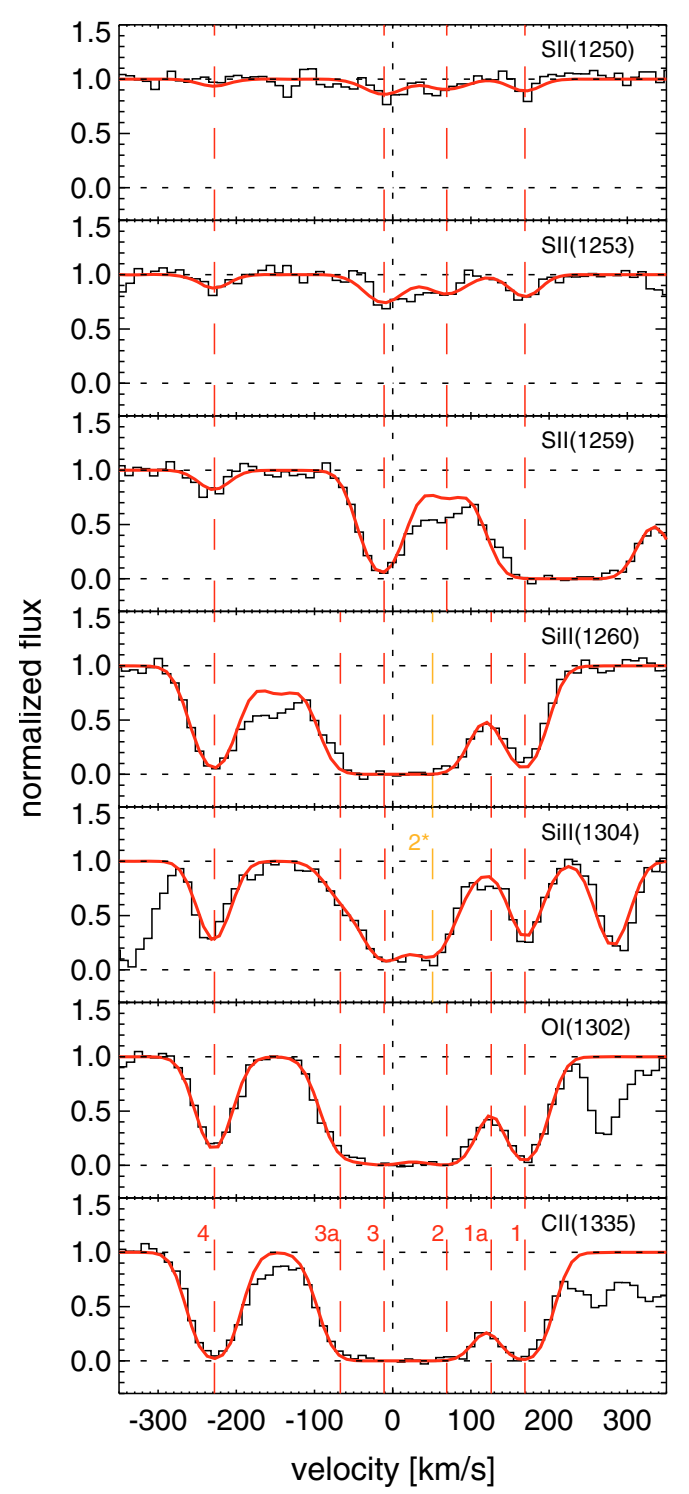

Fig. 1. Fits to the four velocity components (plus two subcomponents) of the resonant absorption transitions in the host galaxy. The dashed red lines indicate the different velocity components as denoted in the bottom panel, the dotted line is the adopted zero velocity corresponding to $z=4.048$. Note that the second component of Si II marked in orange and denoted as $2 *$ is at a slightly different position as for the other lines. The S II $\lambda 1259$ is blended with Si II $\lambda 1260$ and was excluded from the common S II multiplet fit, for the plot of the $\lambda 1259$ line we therefore adopt the column derived from the fit of $\lambda \lambda 1250$ and 1253. The components and their properties are listed in Table 1.

\section{Velocity components in the host galaxy}

The velocity components in the absorption lines span a range of about $400 \mathrm{~km} \mathrm{~s}^{-1}$. The outer components at $169 \mathrm{~km} \mathrm{~s}^{-1}$ (\#1) and $-228 \mathrm{~km} \mathrm{~s}^{-1}$ (\#4) are relatively narrow with $b=15 \mathrm{~km} \mathrm{~s}^{-1}$ (\#2 \& $\# 3$ ), whereas several absorption lines of the two components in between are heavily saturated with $b=25 \mathrm{~km} \mathrm{~s}^{-1}$. In the highest redshift component, which is slightly offset in velocity, the high ionization N V line is detected.

In Paper I we noted the tentative detection of $\mathrm{H}_{2}$ at the redshift of the host galaxy. The position of the $\mathrm{H}_{2}$ transitions are coincident with the redshift of the main absorption component at $v=-11 \mathrm{~km} \mathrm{~s}^{-1}$ which would then be associated with dense

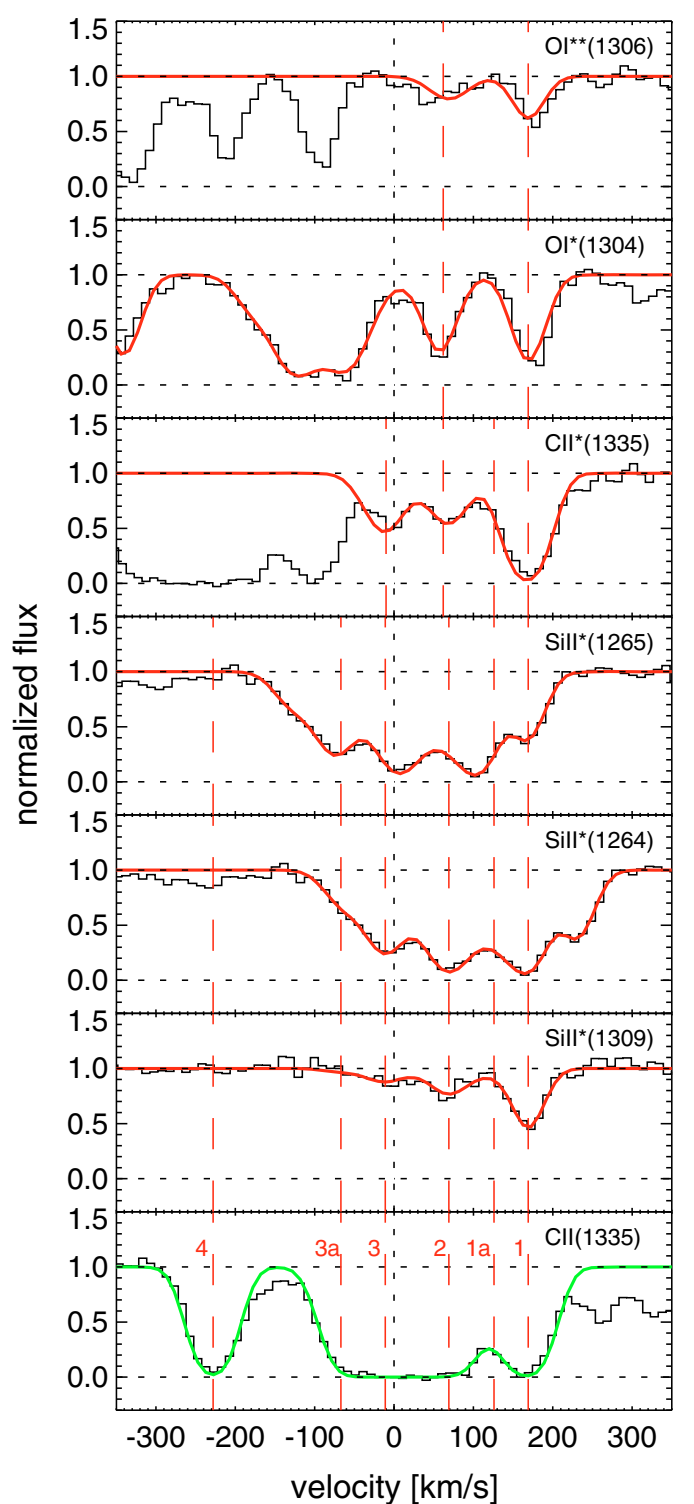

Fig. 2. Same as Fig. 1 for the finestructure lines detected, the bottom panel shows the fit to the C II system for comparison. The finestructure lines only show three or less components. O I* $\lambda 1304$ is blended with Si II $\lambda 1304$ and only the two redmost component have been fitted for both OI** $\lambda 1304$ and OI** $\lambda 1306$. Si II* $\lambda \lambda 1264$ and 1265 and $\mathrm{C}$ II* $\lambda \lambda$ 1335.6, 1335.7 are intrinsically blended and have been fitted together - for clarity, only the fit to $\mathrm{C}_{\mathrm{II}}^{*} \lambda 1335.6$ is shown.

molecular clouds inside the host. All other $\mathrm{H}_{2}$ transitions are be blended with Lyman- $\alpha$ forest absorption lines, so they cannot be identified. The detection of $\mathrm{H}_{2}$ is also consistent with the high metallicity derived for the host of GRB 060206 (Ledoux et al. 2003; Petitjean et al. 2006; Noterdaeme et al. 2008). However, the position of the $\mathrm{H}_{2}$ absorption at the velocity of the main absorption component as seen from the metal line transitions might be the reason we had identified it with $\mathrm{H}_{2}$ transition, which would not have been the case had the absorption occurred at the velocity of one of the weaker components.

\subsection{Finestructure levels}

In Paper I we used the detected finestructure lines to determine the density and temperature for the highest redshift component, 
Table 3. Absorption line fitting of the intervening system at $z=1.48$.

\begin{tabular}{llllllll}
\hline \hline ID & $\begin{array}{l}\lambda_{\text {rest }} \\
{[\AA]}\end{array}$ & comp. & $\begin{array}{l}\lambda_{\text {obs. }} \\
{[\AA]}\end{array}$ & $\begin{array}{l}v_{\text {abs }} \\
{\left[\mathrm{km} \mathrm{s}^{-1}\right]}\end{array}$ & $\begin{array}{l}\log N \\
{\left[\mathrm{~cm}^{-2}\right]}\end{array}$ & $\begin{array}{l}\mathrm{b}_{\text {rest }}\left[\mathrm{km} \mathrm{s}^{-1}\right] \\
{\left[\mathrm{km} \mathrm{s}^{-1}\right]}\end{array}$ & $\begin{array}{l}\log N_{\text {tot }} \\
{\left[\mathrm{cm}^{-2}\right]}\end{array}$ \\
\hline Fe II & 2586 & 1 & 6412.72 & 25 & $13.27 \pm 0.07$ & $19.9 \pm 3.4$ & $13.78 \pm 0.05$ \\
& 2586 & 2 & 6411.51 & -30 & $13.62 \pm 0.07$ & 14 & \\
Fe II & 2600 & 1 & 6446.24 & 25 & $13.27 \pm 0.07$ & $19.9 \pm 3.4$ & $13.78 \pm 0.05$ \\
& 2600 & 2 & 6445.03 & -30 & $13.62 \pm 0.07$ & 14 & \\
Mg II & 2796 & 1 & 6932.60 & 25 & $13.49 \pm 0.08$ & $19.9 \pm 3.4$ & $13.95 \pm 0.08$ \\
& 2796 & 2 & 6931.30 & -30 & $13.77 \pm 0.11$ & 14 & \\
Mg II & 2803 & 1 & 6950.40 & 25 & $13.49 \pm 0.08$ & $19.9 \pm 3.4$ & $13.95 \pm 0.08$ \\
& 2803 & 2 & 6949.09 & -30 & $13.77 \pm 0.11$ & 14 & \\
\hline
\end{tabular}

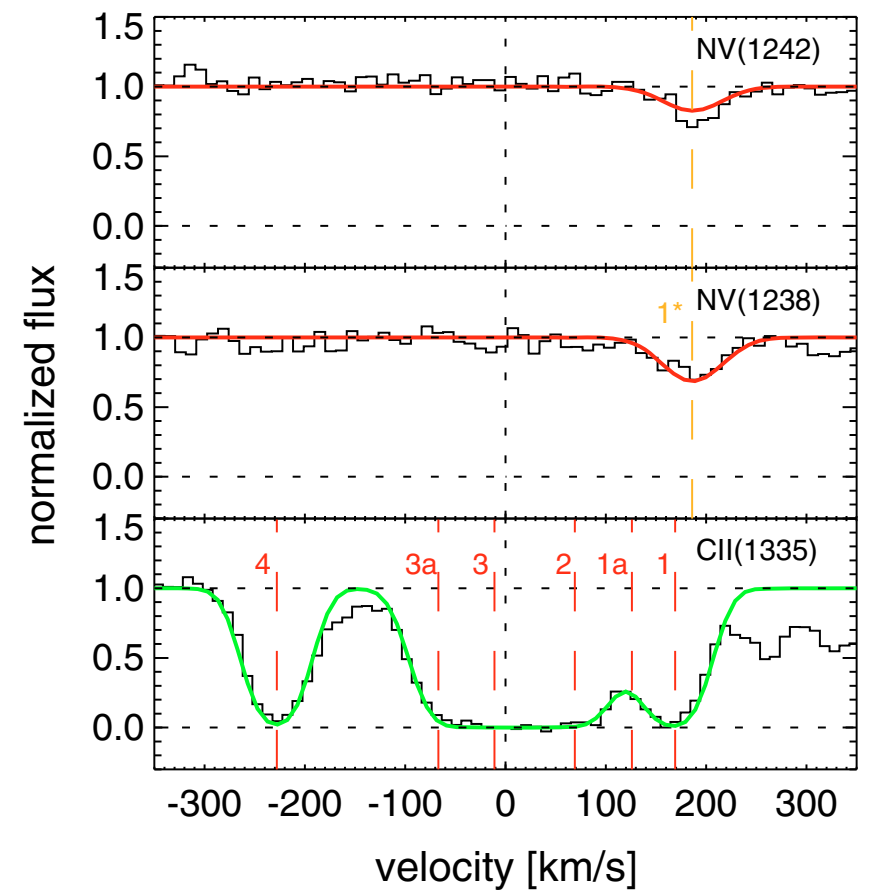

Fig. 3. Fit to the $\mathrm{N} v$ doublet at $\lambda \lambda 1238,1242$ and comparison to the components in $\mathrm{C}$ II. The only component detected, named $1^{*}$, is at a slightly higher redshift than comp. 1 in all other lines which might indicate a different origin in the host galaxy.

assuming that the lines were excited by collisional excitation. However, the VLT/UVES RRM campaign on GRB 060418 (Vreeswijk et al. 2007) has shown that at least some the Fe II and $\mathrm{Ni}$ II finestructure lines in GRB afterglows are excited by indirect UV pumping from the bright GRB and its afterglow, rather than by collisional excitation. This was also put forward by Prochaska et al. (2006) for the spectrum of GRB 051111.

Vreeswijk et al. (2007) measured strong variation with time of the columns of absorption lines and use this to obtain a direct distance determination of the absorbing material with respect to the GRB, by fitting the columns with indirect UV pumping models. Our spectra of GRB 060206 do not have the required temporal and spectral resolution for such an analysis. We can, however, determine approximate properties for the absorbers assuming that indirect UV pumping or collisional excitation is the way that the finestructure levels are populated. Furthermore, the combined WHT spectrum was taken when a significant optical rebrightening occurred in the afterglow light curve (see the light curve by Curran et al. 2007) and UV photons from this

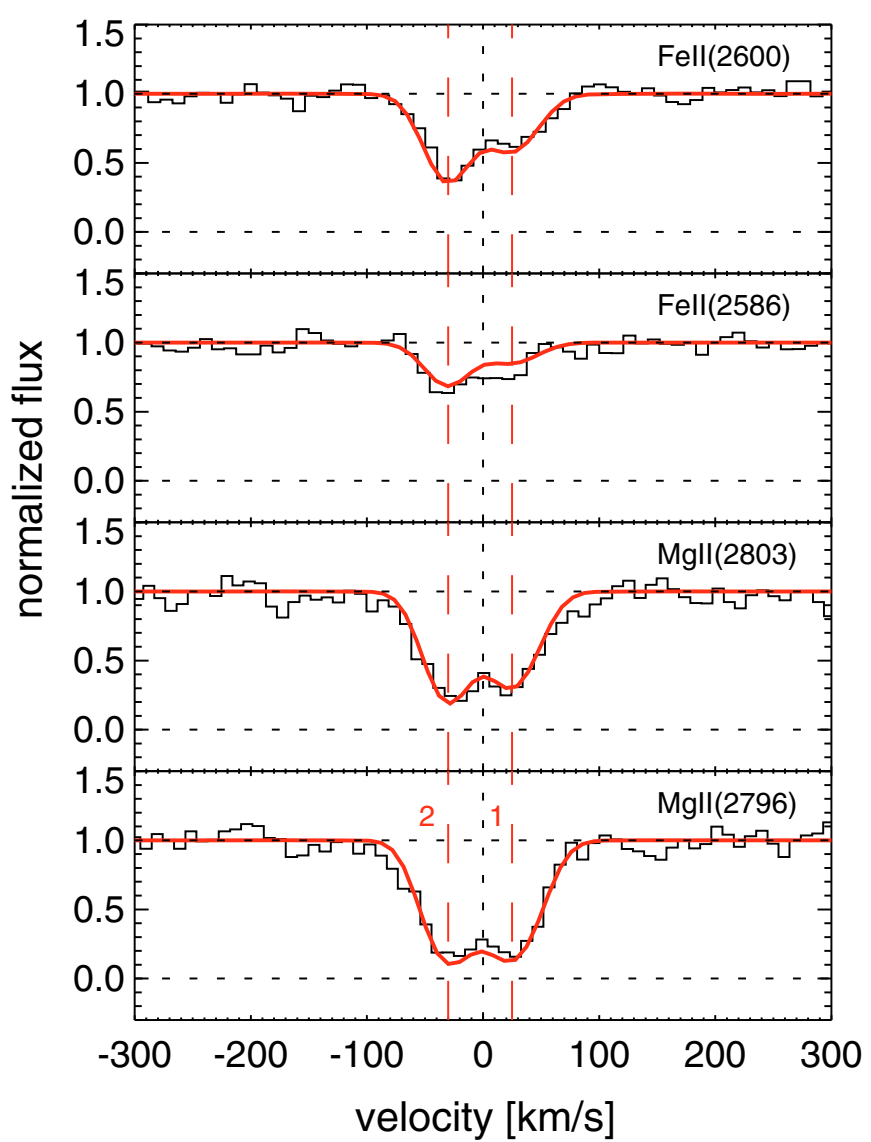

Fig. 4. Fit to the intervening system in the line of sight at redshift $z=1.48$. The dotted lines indicate zero velocity which is the average of the velocity components. The two Mg II and Fe II transitions show two velocity components indicated by the dashed lines which have been fitted as doublets.

rebrightening may have played a significant role in the indirect UV excitation of the transitions. In Table 4 we note the ratios between the finestructure and ground state column densities for the four main components. Due to the uncertainties in the distribution of the column densities between comp. 1 and 1a and 3 and $3 \mathrm{a}$, the densities of the main- and the subcomponent have been added. For the calculation of $n_{\mathrm{e}}$ and $G / G_{0}$, the ratios derived from $\mathrm{Si}$ II*/Si II have been used as they have the smallest errors, according to Prochaska et al. (2006, Figs. 7 and 9).

We can now use these line ratios to estimate the UV radiation field needed to excite the levels, following (Prochaska et al. 2006, Fig. 7), assuming that UV pumping is the dominant 
Table 4. Properties of the four velocity systems observed in the WHT spectrum, see Sect. 5.1.

\begin{tabular}{llllllll}
\hline \hline Comp. & $\begin{array}{l}v_{\text {abs }} \\
{\left[\mathrm{km} \mathrm{s}^{-1}\right]}\end{array}$ & Si II*/Si II & O I*/O I & O I**/O I* $^{* *}$ C II $/$ C II & $\begin{array}{l}n_{\mathrm{e}} \\
{\left[\mathrm{cm}^{-3}\right]}\end{array}$ & $G / G_{0}$ \\
\hline $1+1 \mathrm{a}$ & 148 & $0.596 \pm 0.029$ & $0.07 \pm 0.03$ & $0.195 \pm 0.004$ & $0.23 \pm 0.05$ & $400 \pm 50$ & $3 \pm 0.5 \times 10^{5}$ \\
2 & 69 & $0.115 \pm 0.002$ & $0.005 \pm 0.001$ & $3.24 \pm 0.19$ & $0.07 \pm 0.02$ & $70 \pm 5$ & $6 \pm 0.15 \times 10^{4}$ \\
$3+3 \mathrm{a}$ & -39 & $0.047 \pm 0.001$ & $\ldots$ & $\ldots$ & $<0.003$ & $15 \pm 2$ & $2 \pm 0.15 \times 10^{4}$ \\
4 & -228 & $<0.005$ & $\ldots$ & $\ldots$ & $\ldots$ & $<2$ & $<5 \times 10^{3}$ \\
\hline
\end{tabular}

excitation mechanism for this component. The column density ratio $\left[\mathrm{Si} \mathrm{II}^{*} / \mathrm{Si} \mathrm{II}\right]$ indicates a radiation field intensity for the highest redshift component of $G / G_{0}=3 \times 10^{5}$ (with $G_{0}=1.6 \times 10^{-3} \mathrm{erg} \mathrm{cm}^{-2} \mathrm{~s}^{-1}$ ). Using the ratio [O I*/O I] gives very similar values but with a larger uncertainty. A similar strength of the radiation field was found for the component with the second-highest redshift in the spectrum of GRB 050730 (D'Elia et al. 2007), and is somewhat lower than the value found for GRB 051111 (Prochaska et al. 2006). The required photon field drops to values of $6 \times 10^{4}, 2 \times 10^{4}$ and $\lesssim 10^{4} G / G_{0}$ for the lower redshift absorbers 2,3 and 4, the uncertainties for $G$, however, are quite large (see Prochaska et al. 2006). If all the velocity components in the host are excited through indirect UV pumping by photons of the GRB and afterglow the highest redshift component would be closest to the burst, and the lowest lies furthest away. Fine-structure lines of Si II* were also detected in the spectra of LBGs (Pettini et al. 2002) which might be excited by the UV radiation field by young stars and might therefore be present in any highly starforming galaxy. The decreasing strength of the lines however are in favor of a well confined UV source like a GRB and its afterglow.

In case we assume collisional excitation being the mechanism responsible for the finestructure lines, the required densities are 400, 70, 15 and $<2 \mathrm{~cm}^{-3}$ for the systems 1 to 4 (see Table 4), using the method described in Prochaska et al. (2006, see Fig. 9). These values are comparable to the electron densities found in starforming regions and molecular clouds in our Galaxy and GRB host galaxy starforming regions (e.g. Thöne et al. 2007; Wiersema et al. 2007, and references therein).

We consider indirect UV pumping by the GRB and its afterglow radiation as the most likely scenario for causing the finestructure lines although we cannot exclude that collisional excitation could play a role. The drop in the inferred radiation field from the higher to the lower redshift components and the lack of finestructure absorption in component 4 indicates an increasing distance from the GRB from components 1 to 4 . In the indirect UV pumping scenario we may interpret the decrease in finestructure line excitation with velocity along the line of sight as a distance effect.

No neutral species with ionization potentials lower than hydrogen are observed in the highest redshift absorber (O I has an ionization potential just over 1 Ryd and can therefore be screened by hydrogen), so we cannot set a constraining lower limit to the distance to the GRB for this system through ionization analysis. If we assume that indirect UV pumping by the afterglow of the GRB is exciting the levels in the highest redshift system, we can derive an order of magnitude estimate of the distance to the GRB (see Prochaska et al. 2006) using the value of $G$ found above. From the afterglow light curve (Curran et al. 2007), we calculate the flux received at Earth at the time of the spectrum, using the spectral index derived in Sect. 3 and the restframe frequency of $1.7 \times 10^{15} \mathrm{~Hz}$ as in Prochaska et al. (2006). We find a distance of the highest redshift system to the GRB of order one kpc, which is similar to the more accurate distance found for the absorbing gas through absorption line variability in GRB 060418 (Vreeswijk et al. 2007).

\subsection{N V absorption lines}

Due to its large ionization potential (77.5 to $97.9 \mathrm{eV}), \mathrm{N} \mathrm{V}$ is therefore assumed to arise only in high temperature or shock environments. It has been frequently detected in GRB sightlines (Prochaska et al., submitted) with large column densities of $\log (N)>14 \mathrm{~cm}^{-2}$. They seem to probe cold, narrow gas and often only have small offsets from low ionization and finestructure lines as we also observe for the spectra presented in this paper. Prochaska et al. argue that the N V absorption likely arises from photoionization of the immediate $(<10 \mathrm{pc})$ environment of the GRB through the afterglow, but also shock ionization in starburst winds could in principle play a role and $\mathrm{N} V$ has been observed in LBGs and starbursts (Pettini et al. 2002; Savaglio et al. 2002). Heated gas in the halo traced by other high ionization lines such as O VI, cannot produce the required high column densities, while a connection to shocks from the progenitor wind would imply large offsets which are not observed.

$\mathrm{N} v$ has also been observed in many QSO spectra and in lines of sight towards stars in the LMC (e.g. Caulet 1996) where these lines are however broader than those observed in QSO and high redshift starbursts. Galactic sightlines towards early type stars (e.g. Welsh \& Lallement 2005; Savage et al. 2001), in WolfRayet (WR) star winds and galactic outflows. In the solar neighborhood, it often occurs at the (conductive) interfaces between evaporating hot and cooler interstellar gas (Slavin 1989; Welsh \& Lallement 2005), e.g. at the boundary of a hot bubble formed by $\mathrm{O}$ and $\mathrm{B}$ stars with the neutral ISM, where one would expect to find both high ionization and low ionization species at comparable velocities. The observed high $b$ parameter is consistent with this picture (Savage \& Sembach 1996), but higher as observed for GRB N V absorption and the column density is usually $\log (N)<14 \mathrm{~cm}^{-2}$.

\subsection{The nature of the absorption systems}

Features similar to what we observe in the spectra of GRB 060206 have been discovered in other GRB spectra such as GRB 020813 (Fiore et al. 2005), GRB 021004 (e.g. Fiore et al. 2005), GRB 030329 (Thöne et al. 2007), GRB 050730 (Starling et al. 2005a; Chen et al. 2005; Prochaska et al. 2007), GRB 050820 (Prochaska et al. 2007), GRB 050922C (Piranomonte et al. 2007), GRB 051111 (Penprase et al. 2006; Prochaska et al. 2007), GRB 060218 (Wiersema et al. 2007) and GRB 060418 (Vreeswijk et al. 2007; Prochaska et al. 2007). The velocities of these systems span a wide range from 200 to $3000 \mathrm{~km} \mathrm{~s}^{-1}$ and might have different origins according to their widths, ionization states, and abundance ratios. 
Components with large velocity spreads were sometimes connected to earlier mass losses of the progenitor Wolf-Rayet (WR) star. Van Marle et al. (2005) numerically evaluate a scenario in which the progenitor star wind interacts with circumstellar material, including the mass loss of earlier stages in the progenitor evolution, forming shells of material propagating outwards at a range of velocities. The lower velocity components up to $400 \mathrm{~km} \mathrm{~s}^{-1}$ match the models by Van Marle et al. very well, but we do not detect the expected components at 2000-3000 $\mathrm{km} \mathrm{s}^{-1}$. In the spectra of GRB 021004, these highvelocity components were only detected in C IV and Si IV which are outside the usable range of our WHT spectrum. Furthermore, all components of the GRB 060206 absorption systems show strong low ionization lines which disfavors an origin very close to the burst as the radiation field from the GRB ionizes the surrounding material. Chen et al. (2007) conclude from a sample of high-resolution afterglow spectra that high-velocity components are absent in $80 \%$ of the spectra and the incidence of C IV absorbers along DLA sightlines nonnegligible which makes it more likely that those high-velocity structures arise in intervening galaxies.

Excluding the connection to the immediate environment of the burst, the absorption features must originate in the host galaxy ISM. Systems with small velocity spreads $\left(v<250 \mathrm{~km} \mathrm{~s}^{-1}\right)$ have been found in high-resolution spectroscopic studies on QSO absorbers using mainly Mg II and C IV systems (e.g. Nestor et al. 2005; Churchill et al. 2003; Boksenberg 2003). These systems are often connected to strong Ly $\alpha$ absorption and consist of a saturated central profile and weaker subsystems at higher velocities. Churchill et al. (2003) and Ellison et al. (2003) suggested a rotating disc together with a halo to explain the different systems. However, as shown in Sect.3, the extinction in the line-of-sight to the GRB is consistent with zero, which makes it unlikely that the GRB was situated behind most of the disc material. Also the hydrogen column density of $\log N=20.85 \pm 0.1$ (Paper I) is relatively low for a GRB-DLA (Jakobsson et al. 2006b) implying a position of the GRB inside or at the near end of the galaxy. Furthermore, finestructure lines are usually detected only in the component that dominates the optical depth, associated with the gas inside the galaxy and their redshift has therefore been taken as a proxy for the redshift of the galaxy (Prochaska et al. 2008).

Another possible explanation for those components is absorption by material from a supergalactic wind (Bond et al. 2001). The cumulative supernova explosions within a starforming region can expel gas out into the halo of the galaxy which fragments and produces clumps of material outside the galaxy. These galactic winds have been directly observed in some nearby galaxies (e.g. M82, NGC 3079 and NGC 4945). The inner parts of these superbubbles are relatively hot and show $\mathrm{X}$-ray emission together with optical emission lines, followed by a warm transition phase traced by highly ionized metal absorption lines such as O VI and C IV. The outer shells are colder and show low ionized absorption systems (see e.g. Heckman et al. 2001). Low ionization absorption features in GRB spectra that can be explained by galactic winds have been found in GRB 030329 (Thöne et al. 2007) and GRB 051111 (Penprase et al. 2006). Bond et al. (2001) and Ellison et al. (2003) suggest that the symmetric structures in some $\mathrm{Mg}$ II absorption systems towards QSOs (Bouché et al. 2006) could also be an indication for a galactic-scale outflow scenario.

If we can transform the radiation field derived from the finestructure lines into a distance scale for the different components, assuming that $G \sim 1 / r^{2}$ (see Prochaska et al. 2006) and

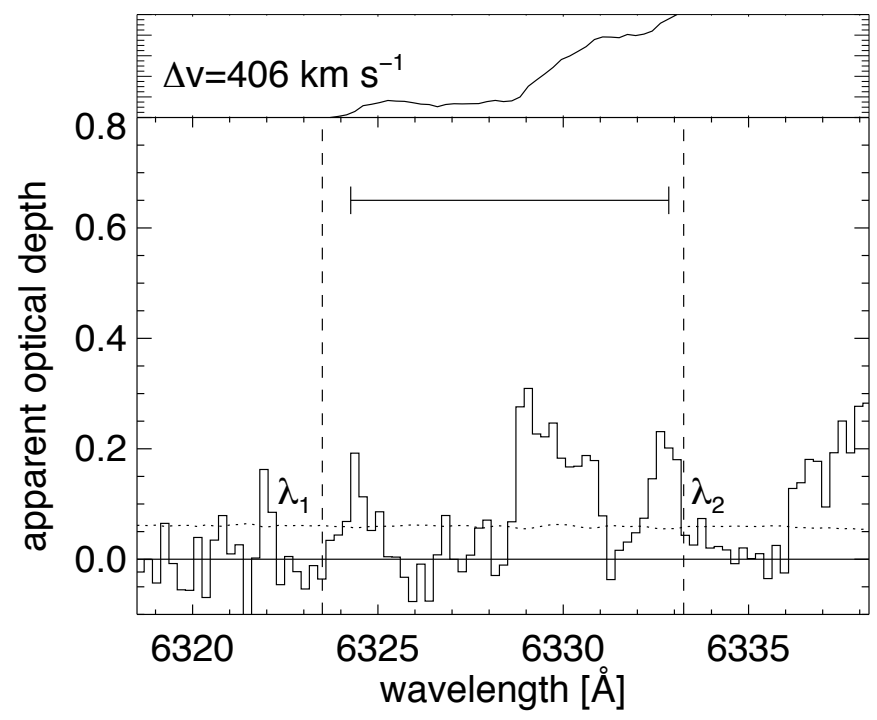

Fig. 5. Apparent optical depth distribution of the $S$ II $\lambda 1253$ system from which we infer a velocity width of $417 \mathrm{~km} \mathrm{~s}^{-1} . \lambda_{1}$ and $\lambda_{2}$ indicate 5 and $95 \%$ of the apparent optical depth distribution. The top panel shows the cumulative EW along the line-of-sight

component 1 to be at $\sim 1 \mathrm{kpc}$ (see Sect. 5.1), component 2, 3 and 4 would then be at distances of $2.2,5.5$ and $>8 \mathrm{kpc}$ from the burst. Comparing this with the velocity spread of $417 \mathrm{~km} \mathrm{~s}^{-1}$, the absorbing systems can hardly be caused by the rotation field in an $0.3 \mathrm{~L}^{*}$ galaxy with a typical size of a few tens of kpc and a rotational velocity of around $150-200 \mathrm{~km} \mathrm{~s}^{-1}$. However, the distances are derived assuming steady-state conditions for the radiation field, which is clearly not the case for a GRB, and can only be considered as rough estimates. Therefore, some of the components could indeed be caused by the gas in the main part of the galaxy whereas others are due to an outflow.

Galactic winds are considered to be the main cause for the metal enrichment of the intergalactic medium. Observing this in the spectra of a burst at $z=4.048$, it shows that these mechanisms started quite early in the history of the Universe. To conclusively discriminate between the possibility of a galactic outflow and absorption within the galaxy, we would need the exact redshift of the galaxy from emission lines as it was possible for GRB 030329 (Thöne et al. 2007). There, the host was a small starburst galaxy with the redshift determined from several emission lines, whereas the Mg I and II absorption systems detected spanning a range of $260 \mathrm{~km} \mathrm{~s}^{-1}$ were mostly blueshifted compared to the host emission lines. This indicates some sort of outflow from that galaxy which was interpreted as a sign of a galactic superwind.

\subsection{Comparison with QSO-DLAs}

To compare the kinematics with QSO-DLAs we follow the procedure of Ledoux et al. (2006) and calculate the line profile velocity width, $\Delta v$, as $c[\lambda(95 \%)-\lambda(5 \%)] / \lambda_{0}$, where $\lambda(5 \%)$ and $\lambda(95 \%)$ are the wavelengths corresponding to, respectively, the five and 95 percentiles of the apparent optical depth distribution, and $\lambda_{0}$ is the first moment (the average) of this distribution (see Fig. 1 of Ledoux et al. 2006). We choose the S II $\lambda 1253$ transition as it is a low-ionization transition and the line is not saturated. The apparent optical depth for the line at the derived velocity width is shown in Fig. 5. We infer a velocity width of $417 \mathrm{~km} \mathrm{~s}^{-1}$, slightly larger than the distribution of DLAs with 


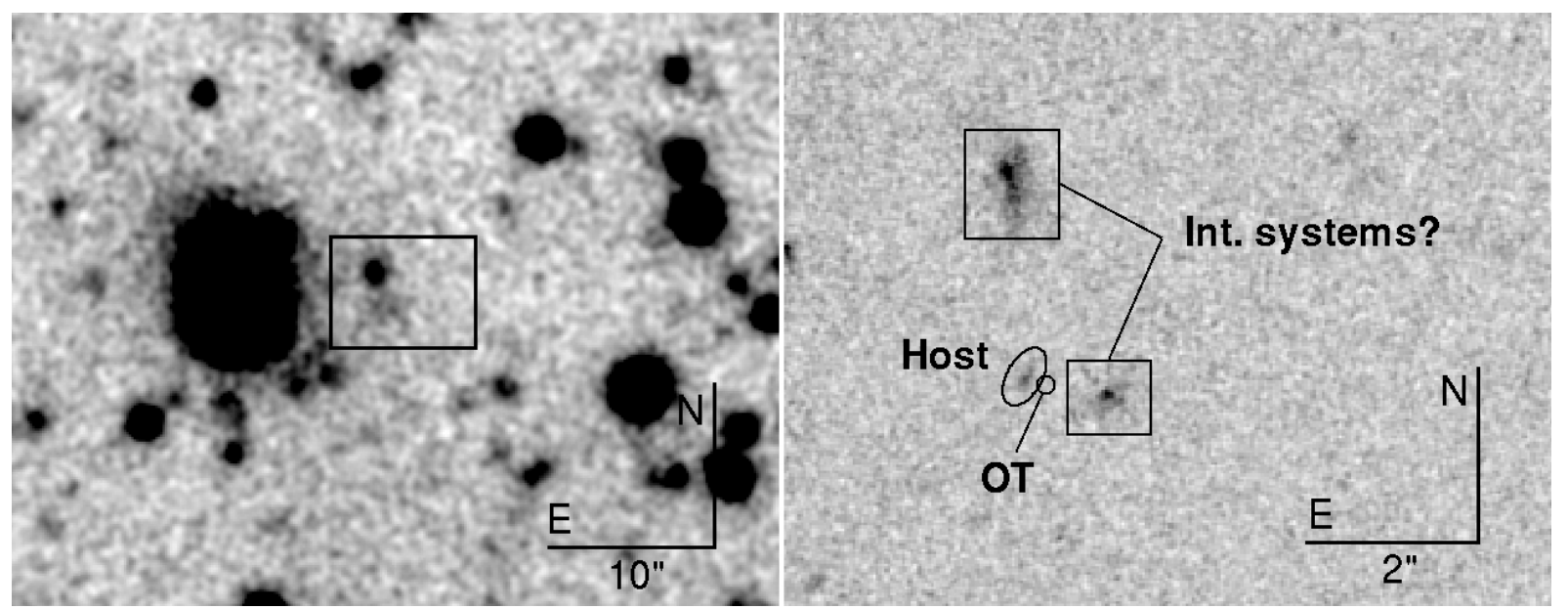

Fig. 6. Left panel: Gemini $r^{\prime}$-band image of the host candidate for GRB 060206. The box marks the cut displayed in the panel to the right which shows an HST images of the host taken with the $F 814 W$ filter. The OT (circle) lies about 0 ! 2 from a faint object (ellipse) which is most likely the host of GRB 060206. The two nearby objects (boxes) are likely candidates for the intervening system where the top one corresponds to the object seen inside the box in the Gemini image.

comparably high metallicities (Fig. 2 in Ledoux et al. 2006; see also Prochaska et al. 2007).

This indicates that the kinematics of GRB absorbers is similar to that of QSO-DLAs and hence that the immediate environment of the GRB, e.g. the wind from the progenitor star, is not strongly dominating the kinematics and metallicity of the system. This is consistent with the recent findings of Vreeswijk et al. (2007) and Watson et al. (2007) that the GRB absorbers probe gas that is more than several kpc away from the GRB site. It also supports our suggestion that the structures observed come from material in the galaxy or from a galactic outflow far away from the GRB.

\section{The host in emission}

In the deep $r^{\prime}$ Gemini images of $2700 \mathrm{~s}$, we find a candidate for the host galaxy with $25 \pm 0.2 \mathrm{mag}(\mathrm{AB})$. The host was not detected and the $3 \sigma$ detection limit at the coordinates of the host is $H>20.6$ mag (Vega). The right panel of Fig. 6 shows the Gemini $r^{\prime}$ band image. In this image, the host appears to have a rather low surface brightness with two slightly brighter knots where the afterglow position falls on top of one of them.

The HST image resolves the two knots of the possible host from the Gemini images into two objects. One of them is a faint galaxy underlying this position, with the burst offset roughly 0.2 from its centroid (see Fig. 6). We assume that this is the host galaxy of GRB 060206. This galaxy lies close to two other galaxies, of which the one to the west was blended blended with the actual host galaxy at the resolution of our ground-based Gemini image. One of these nearby galaxies may well represent the system seen as the foreground absorbers within our afterglow spectroscopy. The magnitude of the galaxy, measured in an aperture of radius 0.2 is $F 814 W_{A B}=27.48 \pm 0.19$ mag.

The detection of a GRB host galaxy at such high redshift is relatively rare: so far, only three hosts for long GRBs with afterglows at $z>3$ have been found - GRB $971214(z=3.42)$ (Kulkarni et al. 1998), GRB000131 $(z=4.5)$ (Fruchter et al. 2006) and GRB $030323(z=3.372)$ (Vreeswijk et al. 2004) despite the fact that 26 GRBs are known with $z>3$ so far. At a redshift of $z=4.048, F 814 W_{A B}=27.48$ corresponds to an absolute magnitude of -20.34 mag or roughly $0.3 L^{*}$, not taking into account the redshift evolution of the luminosity function.
The nondetection of the host galaxy in deep $H$ band imaging performed with the CAHA $3.5 \mathrm{~m}$ telescope is consistent with a starforming host with the measured $F 814 W_{A B}$ magnitude.

\section{An intervening system at $z=1.48$}

\subsection{Properties of the intervening absorber}

In addition to the systems intrinsic to the GRB redshift, we detect an intervening absorber at $z=1.48$. It contains two velocity systems of the two doublets Mg II $\lambda \lambda 2796,2803$ and Fe II $\lambda \lambda 2586$, 2600 with a separation of $\sim 50 \mathrm{~km} \mathrm{~s}^{-1}$. In Paper I, we described a second absorbing system at $z=2.259$. In the reanalysis performed in this paper, we conclude, however, that this system is not real.

In a sample of QSO absorbers, Churchill et al. (2003) find that the column densities of $\mathrm{Mg}$ II and Fe II correlate linearly. The column densities we find for both velocity systems fall within 1 sigma of the linear correlation of Churchill et al. (2003). The $b$ values we find are very high for both components compared with the sample of Churchill et al. (2003), pointing either to very high temperatures or to more velocity components present that we cannot resolve at our intermediate resolution. The latter is supported by the correlation that Churchill et al. (2003) find between the restframe equivalent width of the Mg II $\lambda 2796$ line and the number of velocity components in the absorber, which predicts a higher number of velocity components $(\sim 10)$ than the two we find in the WHT spectra.

\subsection{Variability of the intervening system?}

Hao et al. (2007) claim significant variability in the equivalent widths (EWs) of the Mg II doublet and the Fe II $\lambda 2600$ line, in the time interval between $4.13 \mathrm{~h}$ and $7.63 \mathrm{~h}$ after the burst, covered by 7 low-resolution spectra. They fit the lines with Gaussian profiles, and report variation in both the equivalent widths and the relative velocities of the lines.

The two WHT spectra were taken earlier than those reported in Hao et al. (2007) with mid-points 1.73 and $2.01 \mathrm{~h}$ after burst. The spectral resolution and S/N of the WHT spectra are significantly better than those used by Hao et al. (2007). Both the Fe II and $\mathrm{Mg}$ II lines can be split into two separate velocity 


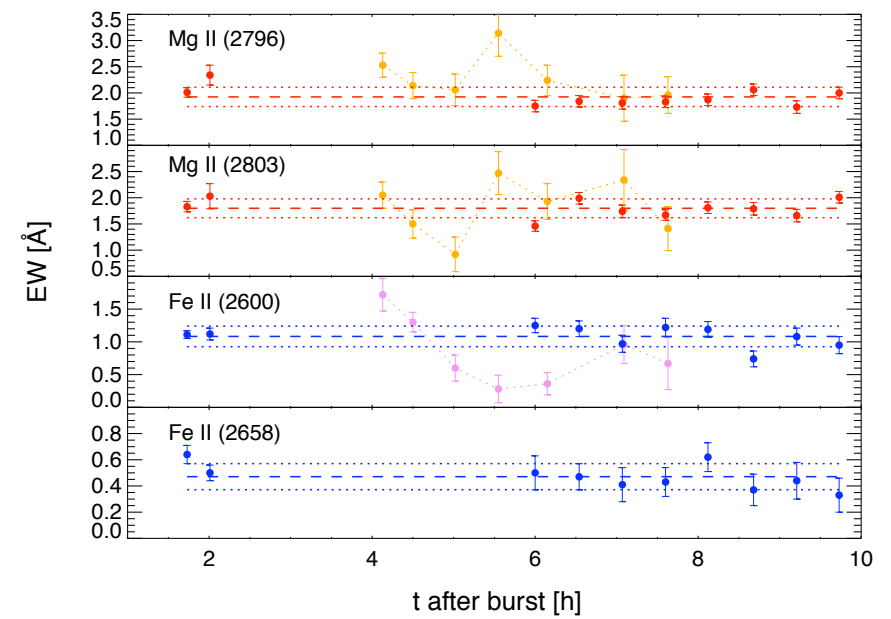

Fig. 7. Equivalent widths measured from the WHT and Subaru spectra (red and blue points) and values from Hao et al. (2007) (orange and violet). In addition, we plot the mean of the WHT and Subaru points (dashed lines) and the $1 \sigma$ errors (dotted lines) of the mean. Times given are the midtimes of the observations. The plots shows no trend in the evolution of the EW with time as claimed in Hao et al. (2007) and our values are consistent with the mean mostly within $1 \sigma$.

components which would be unresolved in low-resolution spectra, but we measure EWs of these two systems combined to compare directly with the Hao et al. (2007) values. We furthermore analyzed the low-resolution Subaru data, taken around the same time as the spectra reported in Hao et al. (2007).

The EW measurements of the Fe II $\lambda 2600$ Aline from both the WHT and the Subaru data show no variations within the errors and are clearly in disagreement with the strong variability in Hao et al. (2007). The measurement of the Mg II lines is complicated by the fact that they are superimposed on the atmospheric $B$ band and the continuum can therefore not be reliably determined. Hao et al. (2007) removed the absorption band before fitting the $\mathrm{Mg}$ II lines but note that they had a correction of about $25 \%$ of the variation in absorption band caused by the varying airmass during their observations. The apparent variability in the line shape and strength in their $\mathrm{Mg}$ II lines is therefore likely due to inadequate removal of the atmospheric band. We also note that the similar EW of the two components of the $\mathrm{Mg}$ II doublet which should have a ratio of 1:2 indicates that these lines are saturated.

For a comparison between the WHT and the Subaru values and the ones derived by Hao et al. (2007), see Fig. 7. The EW measurements are listed in Table 5. The EWs have been measured in the WHT spectra, where we analyzed the two $900 \mathrm{~s}$ exposures separately and in the Subaru spectra retrieved from the archive. $E W \mathrm{~s}$ are in the observer frame.

\subsection{Identifying the absorber in emission}

Detecting intervening galaxies in emission is complicated in the case of QSOs, but the transience of GRBs makes them more suitable to detect intervening systems in emission once the afterglow has faded. While intervening systems can produce very clear absorption systems, the identification of specific absorbers with individual galaxies is difficult (e.g. Vreeswijk et al. 2003; Ellison et al. 2006). The Gemini and HST images show several galaxies within a radius of 5 arcsec, with the two most promising candidates $\sim 1$ and $\sim 2.5$ arcsec from the host which corresponds
Table 5. Measurements of the intervening system lines which were reported to vary by Hao et al. (2007).

\begin{tabular}{llllll}
\hline \hline Spec. & $t$ & $\mathrm{Fe} \mathrm{II}_{2658}$ & $\mathrm{Fe} \mathrm{II}_{2600}$ & $\mathrm{Mg} \mathrm{I}_{2796}$ & $\mathrm{Mg} \mathrm{I}_{2803}$ \\
& {$[\mathrm{~h}]$} & $E W[\AA]$ & $E W[\AA]$ & $E W[\AA]$ & $E W[\AA]$ \\
\hline WHT & 1.73 & $0.64 \pm 0.07$ & $1.11 \pm 0.06$ & $2.01 \pm 0.09$ & $1.83 \pm 0.10$ \\
WHT & 2.01 & $0.50 \pm 0.06$ & $1.12 \pm 0.09$ & $2.34 \pm 0.19$ & $2.03 \pm 0.24$ \\
\hline Sub. & 6.00 & $0.50 \pm 0.13$ & $1.25 \pm 0.11$ & $1.75 \pm 0.11$ & $1.46 \pm 0.10$ \\
Sub. & 6.54 & $0.47 \pm 0.10$ & $1.20 \pm 0.12$ & $1.84 \pm 0.11$ & $1.99 \pm 0.11$ \\
Sub. & 7.07 & $0.41 \pm 0.13$ & $0.97 \pm 0.13$ & $1.81 \pm 0.12$ & $1.74 \pm 0.12$ \\
Sub. & 7.60 & $0.43 \pm 0.11$ & $1.22 \pm 0.14$ & $1.83 \pm 0.11$ & $1.67 \pm 0.10$ \\
Sub. & 8.12 & $0.62 \pm 0.11$ & $1.19 \pm 0.12$ & $1.87 \pm 0.11$ & $1.81 \pm 0.11$ \\
Sub. & 8.68 & $0.37 \pm 0.12$ & $0.74 \pm 0.12$ & $2.06 \pm 0.11$ & $1.79 \pm 0.12$ \\
Sub. & 9.21 & $0.44 \pm 0.14$ & $1.08 \pm 0.13$ & $1.73 \pm 0.12$ & $1.66 \pm 0.12$ \\
Sub. & 9.73 & $0.33 \pm 0.13$ & $0.95 \pm 0.13$ & $2.00 \pm 0.11$ & $2.01 \pm 0.11$ \\
\hline
\end{tabular}

to distances of $\sim 8$ and $21 \mathrm{kpc}$ at $z=1.479$. Photometry of those two galaxies yield magnitudes of $F 814 W_{A B}=26.1 \pm 0.1$ for the one to the right, blended with the host in the Gemini images, and $F 814 W_{A B}=24.7 \pm 0.1$ or $r_{A B}^{\prime}=23.9 \pm 0.1$ for the one north of the host.

At $z=1.479$, the GMOS $r^{\prime}$ band and HST F814W filter correspond to restframe $2540 \AA$ and $3360 \AA$ and probe restframe UV emission. We estimate the fluxes of the galaxies 1 and 2.5 arcsec north of the host at $\sim 2800 \AA$ to be $\sim 0.4$ and $\sim 1.2 \mu \mathrm{Jy}$, which corresponds to UV star formation rates of $\sim 8$ and $25 M_{\odot} \mathrm{yr}^{-1}$, not corrected for extinction, using the conversion from Kennicutt (1998). The non-detection of the host or absorber in the $H$ band is consistent with the intervening absorber candidate being a blue starforming galaxy.

Correlations between parameters of the $\mathrm{Mg}$ II absorption lines and the galaxy that produces them has been studied extensively for QSO absorbers. Recently, Zibetti et al. (2007) used the large dataset of the SDSS to correlate the QSO Mg II absorber properties with the light of SDSS galaxies at a range of impact parameters (from 10-200 kpc) and found that stronger absorbers can be related to bluer galaxies. The restframe EW of $2.5 \AA$ of $\mathrm{Mg}$ II $\lambda 2796$ clearly places it in the category of the strong $\mathrm{Mg}$ II absorbers (though the definition of a strong absorber varies from author to author, but we assume $W_{0}(2796) \simeq 1 \AA$ ), which are generally associated with relatively bright galaxies, with luminosities from $\sim 0.1$ to several $L_{*}$. For two other GRB hosts, GRB 021004 and GRB 020813, candidate host galaxies for intervening systems have recently been discovered which seem to lie within a few tens of kpc from the GRB afterglow (Henriksen et al. in prep.). Also GRB 030429 has a tentative detection of an emission line from the host of the intervening system in the afterglow spectrum (Jakobsson et al. 2004) and the intervening absorber in the GRB 020405 spectra could be associated with a neighboring galaxy with the detection of $\mathrm{O}$ II and $\mathrm{O}$ III at the same redshift (Masetti et al. 2003).

Ellison (2006) show that the velocity spread of the Mg II absorber in combination with the restframe equivalent width (the $D$ parameter) supplies an efficient means to select possible DLAs. We find $D \sim 8$ for our absorber, making it likely that this absorber is a DLA which can be probed by Lyman $\alpha$, which is however still in the UV at $z=1.48$ and could not be observed. The blue, luminous and rapidly starforming galaxy very nearby the GRB host fits in this picture.

We note that comparison with QSO absorber samples may be further complicated by the recently established over-abundance 
of $\mathrm{Mg}$ II absorbers on GRB lines of sight compared to QSO sightlines (Prochter et al. 2006), for which the cause is yet unclear. This difference is not present in line of sight detections of C IV, for reasons not fully understood (Sudilovsky et al. 2007; Tejos et al. 2007).

\section{Conclusions}

In this paper we have presented the full set of detected absorption lines in the medium-resolution spectrum from WHT, as well as host galaxy observations with deep imaging in $r^{\prime}$ and $H$ band, using Gemini North and the CAHA $3.5 \mathrm{~m}$ telescope. The spectra show at least four main velocity components at the GRB redshift, spread over a range of $\sim 400 \mathrm{~km} \mathrm{~s}^{-1}$. For each component, we detect a series of absorption lines, including several species of finestructure lines for 3 of the 4 components. Those finestructure lines are not found in QSO absorbers, but seem abundant in GRB lines-of-sight (e.g. Vreeswijk et al. 2004; Starling et al. 2005b; Prochaska et al. 2006; Fynbo et al. 2006; Kawai et al. 2006; Penprase et al. 2006). We measured their column densities through Voigt profile fitting, which is complicated by the blending of the lines of different velocity systems; for every velocity component, however, a diagnostic set of absorption lines can be measured.

The four velocity components show decreasing occupation of their excited finestructure levels with decreasing redshift. The column density ratios of $[\mathrm{Si}$ II*/Si II] indicate that lines in the component with the highest redshift are most likely excited through indirect UV pumping by a strong photon field equivalent to $\sim 3 \times 10^{5} G / G_{0}$, produced by the GRB and its afterglow. The powerful rebrightening of the afterglow (Woźniak et al. 2006; Monfardini et al. 2006; Stanek et al. 2007) may play an important role in this excitation. If indirect UV pumping through the afterglow is the source of excitation, the highest redshift absorber is about of one kpc away from the burst and the other components subsequently at $\sim 2, \sim 5$, and $>8 \mathrm{kpc}$. The similarities of the highest redshift component with the values for GRB 050730 and GRB 051111 and the direct detection of absorption line variability of $\mathrm{Fe} \mathrm{II}^{*}$, Ni II ${ }^{*}$, but not $\mathrm{Si} \mathrm{II}^{*}$, in GRB 060418 make an origin of the lines through indirect UV pumping likely. From the finestructure level analysis that provides the relative positions of the velocity components with respect to the GRB, the velocity spread and the low ionization state of the medium, a starburst wind seems an appealing scenario to cause these absorption features. Their nature could be clarified through high-resolution (space-based) imaging and emission line spectroscopy of the host.

The possible host galaxy is detected at $F 814 W_{A B}=27.48 \pm$ 0.19 mag and an upper limit of $H \geq 20.6(3 \sigma)$ is achieved, which agrees with the spectral energy distribution of a blue starforming galaxy at that redshift. In the image we find several galaxies close to the afterglow position, the closest ones at distances of 1 and 2 .'5. In the spectrum we detect an intervening absorber at $z=1.48$ showing two strong $\mathrm{Mg}$ II and Fe II absorption components that are likely caused by that bright galaxy. This galaxy is not detected in the $H$ band, consistent with it being a spiral or irregular galaxy, which is also suggested from the absorption line properties. Hao et al. (2007) claim significant variability in the equivalent width of the Mg II doublet and the Fe II $\lambda 2600$ line of this absorber. The WHT spectra taken earlier and Subaru spectra taken during the observations of Hao et al. (2007) do not confirm the variability in equivalent width of these lines or the trend in the variability as a function of time.
The high-resolution spectroscopy of GRB 060206 was taken with WHT, demonstrating that these detailed line analyses are clearly possible with $4 \mathrm{~m}$ class telescopes when a bright afterglow presents itself and intermediate or high-resolution spectroscopy is possible.

Acknowledgements. We thank the observers and ING staff for performing the reported observations. CT wants to thank Jason Prochaska and Miroslava Dessauges-Zavadsky for the unpublished version of their paper on NV detections in GRB sightlines.

The Dark Cosmology Centre is funded by the Danish National Research Foundation. KW, PC, and RW thank NWO for support under grant 639.043.302. The authors acknowledge benefits from collaboration within the EU FP5 Research Training Network "Gamma-Ray Bursts: An Enigma and a Tool" (HPRN-CT-2002-00294). This study is supported by the Spanish Ministry of Science through research projects ESP2005-07714-C03-03 and AYA2004-01515.

Based on observations made with the Nordic Optical Telescope, operated on the island of La Palma jointly by Denmark, Finland, Iceland, Norway, and Sweden and with the William Herschel Telescope, in the Spanish Observatorio del Roque de los Muchachos of the Instituto de Astrofísica de Canarias. Based on observations obtained at the Gemini Observatory, which is operated by the Association of Universities for Research in Astronomy, Inc., under a cooperative agreement with the NSF on behalf of the Gemini partnership: the National Science Foundation (USA), the Particle Physics and Astronomy ResearchCouncil (UK), the NationalResearchCouncil (Canada), CONICYT (Chile), the Australian Research Council (Australia), CNPq (Brazil), and CONICET (Argentina) and on observations carried out at the Centre Astronóminco Hispano Alemán (CAHA) at Calar Alto, operated jointly by the Max-Planch Institut für Astronomie and the Instituto de Astrofísica de Andalucía (IAA-CSIC). Based in part on data collected at Subaru Telescope and obtained from the SMOKA, which is operated by the Astronomy Data Center, National Astronomical Observatory of Japan.

\section{References}

Adelberger, K. L., Steidel, C. C., Shapley, A. E., \& Pettini, M. 2003, ApJ, 584, 45 Asplund, M., Grevesse, N., Sauval, A. J., Allende Prieto, C. \& Kiselman, D. 2004, A\&A, 417, 751

Baba, H., et al. 2002, ADASS XI, ed. D. A. Bohlender, D. Durand, \& T. H. Handley, ASP Conf. Ser., 281, 298

Boksenberg, A., Sargent, W. L. W., \& Rauch, M. 2003, ApJS, submitted, [arXiv: astro-ph/0307557]

Bond, N. A., Churchill, C. W., Charlton, J. C., \& Vogt, S. S. 2001, ApJ, 557, 761 Bouché, N., Murphy, M. T., Péroux, C., Csabai, I., \& Wild, V. 2006, MNRAS, 371,495

Bromm, V., \& Loeb, A., 2006, ApJ, 642, 382

Burrows, D. N., Hill, J. E., Nousek, J. A., et al. 2005, Space Sci. Rev., 120, 165

Caulet, A. 1996, Proceedings "Science with the Hubble Space Telescope - II", 481

Cenko S. B., Berger, E., Djorgovski, S. G., Mahabal, A. A. \& Fox, D. B. 2006, GCN Circ., 5155

Chen, H. W., Prochaska, J. X., Bloom, J. S., \& Thompson, I. B. 2005, ApJ, 634, L25

Chen, H.-W., Prochaska, J. X., Ramirez-Ruiz, E., et al. 2007, ApJ, 663, 420

Churchill, C. W., Vogt, S. S., \& Charlton, J. C. 2003, AJ, 125, 98

Ciardi, B., \& Loeb, A. 2000, ApJ, 540, 687

Cool, R. J., Eisenstein, D. J., Hogg, D. W., et al. 2006, GRB Coordinates Network, 4695

Curran, P. A., van der Horst, A. J., Wijers, R. A. M. J., et al. 2007, MNRAS, 381,65

Daigne F., Rossi E. M., \& Mochkovitch R. 2006, MNRAS, 372, 1034

D'Elia, V., Fiore, F., Meurs, E. J. A., et al. 2007, A\&A, 467, 629

Ellison, S. L. 2006, MNRAS, 368, 335

Ellison, S. L., Malln-Ornelas, G., \& Sawicki, M. 2003, ApJ, 589, 709

Ellison, S. L., Vreeswijk, P.M., Ledoux, C., et al. 2006, MNRAS, 372, 38

Fiore, F., D’Elia, V., Lazzati, D., et al. 2005, ApJ, 624, 853

Fontana, E., \& Ballester, P. 1995, ESO Messenger 80, 37

Fruchter, A. S., Levan, A. J., Strolger, L., et al. 2006, Nature, 441, 463

Fynbo, J. P. U., Starling, R. L.C., Ledoux, C., et al. 2006, A\&A, 451, L47

Fynbo, J. P. U., Prochaska, J. X., Sommer-Larsen, J., Dessauges-Zavadsky, M., \& Møller, P. 2008, ApJ, submitted, [arXiv:0801.3273]

Gehrels, N., Chincarini, G., Giommi, P., et al. 2004, ApJ, 611, 1005

Haislip, J. B., Nysewander, M. C., Reichart, D. E., et al. 2006, Nature, 440, 181

Hao, H., Stanek, K. Z., Dobrzycki, A., et al. 2007, ApJ, 659, 99

Heckman, T. M. 2001, in Gas and Galaxy Evolution, ed. J. E. Hibbard, M. Rupen, \& J. H. van Gorkom (San Francisco: ASP) ASP Conf. Proc., 240 
Jakobsson, P., Fynbo, J. P. U., Hjorth, J. et al. 2004, A\&A, 427, 785

Jakobsson, P., Björnsson G., Fynbo, J. P. U., et al. 2005, MNRAS, 362, 245

Jakobsson, P., Levan A., Fynbo, J. P. U., et al. 2006a, A\&A, 447, 897

Jakobsson, P., Fynbo, J. P. U., Ledoux, C. et al. 2006b, A\&A, 460, L13

Kawai, N., Kosugi, G., Aoki, K., et al. 2006, Nature, 440, 184

Kennicutt, R. C. 1998, ARA\&A, 36, 189

Kulkarni S. R., Djorgovski, S. G., Ramaprakash, A. N., et al. 1998, Nature, 393, 35

Ledoux, C., Petitjean, P., \& Srianand, R. 2003, MNRAS, 346, 209

Ledoux, C., Petitjean, P., Fynbo, J. P. U., et al. 2006, A\&A, 457, 71

Masetti, N., Palazzi, E., Pian, E., et al. 2003, A\&A, 404, 465

Monfardini, A., Kobayashi, S., Guidorzi, C., et al. 2006, ApJ, 648, 1125

Nestor, D.B., Turnshek, D. A., \& Rao, S. M. 2005, ApJ, 628, 637

Noterdaeme, P., Ledoux, C., Petitjean, P., \& Srianand, R. 2008, A\&A, 481, 327

Penprase, B. E., Berger, E., Fox, D. B., et al. 2006, ApJ, 646, 358

Petitjean, P., Ledoux, C., Noterdaeme, P., \& Srianand, R. 2006, A\&A, 456, L9

Pettini, M., Rix, S. A., Steidel, C. C., et al. 2002, ApJ, 569, 742

Piranomonte, S., D'Elia, V., Ward, P., Fiore, F., \& Meurs, E. J. A. 2007,

Proceedings of the Swift-Venice 2006 meeting to be published by Il Nuovo Cimento, [arXiv: astro-ph/0701563]

Prochaska, J. X., Chen, H.-W., \& Bloom, J. S. 2006, ApJ, 648, 95

Prochaska, J. X., Chen, H.-W., Dessauges-Zavadsky, M., \& Bloom, J. S. 2007, ApJS, 168, 231

Prochaska, J. X., Chen, H.-W., Wolfe, A. M., et al. 2008, ApJ, 672, 59

Prochter, G. E., Prochaska, J. X., Chen, H.-W., et al. 2006, ApJ, 648, 93

Ruiz-Velasco, A. E., Swan, H., Troja, E., et al. 2007, ApJ, 669, 1

Savage, B. D., \& Sembach, K. R. 1996, ARA\&A, 34, 279
Savage, B. D., Meade, M. R., \& Sembach, K. R. 2001, ApJS, 136, 631 Savaglio, S. 2006, NJoP, 8, 195

Savaglio, S., Panagia, N., \& Padovani, P. 2002, ApJ, 567, 702

Silva, A. I., \& Viegas, S. M. 2002, MNRAS, 329, 135

Slavin, J. D. 1989, ApJ, 346, 71

Stanek, K. Z., Dai, X., Prieto, J. L., et al. 2007, ApJ, 654, L21

Starling, R. L. C., Wijers, R. A. M. J., Hughes, M. A., et al. 2005a, MNRAS, 360,305

Starling, R. L. C., Vreeswijk, P. M., Ellison, S. L., et al. 2005b, A\&A, 442, L21

Starling, R. L. C., Wijers, R. A. M. J., Wiersema, K., et al. 2007, ApJ, 661, 787

Sudilovsky, V., Savaglio, S., Vreeswijk, P. M., et al. 2007, ApJ, 669, 741

Tejos, N., Lopez, S., Prochaska, J. X., et al. 2007, ApJ, 671, 622

Tanvir, N. R., \& Jakobsson, P. 2007, Proceedings of the Royal Society Discussion meeting on Gamma-ray Bursts, September 18-20, 2006, Philos. Trans. Roy. Soc. A, 365, 1377

Thöne, C. C., Greiner, J., Savaglio, S., \& Jehin, E. 2006, ApJ, 672, 621

Tremonti, C., Heckman, T. M, Kauffmann, G. et al. 2004, ApJ, 613, 898

Van Marle, A. J., Langer, N., \& García-Segura, G. 2005, A\&A, 444, 837

Vreeswijk, P. M., Møller, P., \& Fynbo, J. P. U. 2003, A\&A, 409, 5

Vreeswijk, P. M., Ellison, S. L., Ledoux, C., et al. 2004, A\&A, 419, 927

Vreeswijk, P. M., Ledoux, C., Smette, A., et al. 2006, A\&A, 468, 83

Watson, D., Hjorth, J., Fynbo, J. P. U., et al. 2007, ApJ, 660, L101

Welsh, B. Y., \& Lallement, R. 2005, A\&A, 436, 615

Wiersema, K., Savaglio, S., Vreeswijk, P. M., et al. 2007, A\&A, 464, 529

Woźniak, P. R., Vestrand, W. T., Wren, J. A., et al. 2006, ApJ, 642, L99

Zibetti, S., Ménard, B., Nestor, D. B., et al. 2007, ApJ, 658, 151 